\title{
Impact of Rumors and Misinformation on COVID-19 in Social Media
}

\author{
Samia Tasnim ${ }^{1}$, Md Mahbub Hossain', Hoimonty Mazumder ${ }^{2}$ \\ ${ }^{1}$ Department of Health Promotion and Community Health Sciences, Texas A\&M School of Public Health, College Station, TX, USA; ${ }^{2}$ Save the Chil- \\ dren, Dhaka, Bangladesh
}

The coronavirus disease 2019 (COVID-19) pandemic has not only caused significant challenges for health systems all over the globe but also fueled the surge of numerous rumors, hoaxes, and misinformation, regarding the etiology, outcomes, prevention, and cure of the disease. Such spread of misinformation is masking healthy behaviors and promoting erroneous practices that increase the spread of the virus and ultimately result in poor physical and mental health outcomes among individuals. Myriad incidents of mishaps caused by these rumors have been reported globally. To address this issue, the frontline healthcare providers should be equipped with the most recent research findings and accurate information. The mass media, healthcare organization, community-based organizations, and other important stakeholders should build strategic partnerships and launch common platforms for disseminating authentic public health messages. Also, advanced technologies like natural language processing or data mining approaches should be applied in the detection and removal of online content with no scientific basis from all social media platforms. Furthermore, these practices should be controlled with regulatory and law enforcement measures alongside ensuring telemedicine-based services providing accurate information on COVID-19.

Key words: COVID-19, Misinformation, Health impacts, Social media

Coronavirus disease 2019 (COVID-19) is a respiratory tract illness resulting from infection with severe acute respiratory syndrome coronavirus 2 (SARS-CoV-2); in recent months, COVID-19 has become a global pandemic, posing a major public health challenge for the health systems of many nations. Simultaneously, numerous rumors, pieces of misinformation, and hoaxes have appeared on several social media platforms

Received: March 28, 2020 Accepted: April 2, 2020

Corresponding author: Samia Tasnim, MD

Department of Health Promotion and Community Health Sciences, Texas A\&M School of Public Health, 212 Adriance Lab Road, College

Station, TX 77843, USA

E-mail: tasnim@tamu.edu

This is an Open Access article distributed under the terms of the Creative Commons Attribution Non-Commercial License (https://creativecommons.org/licenses/bync/4.0// which permits unrestricted non-commercial use, distribution, and reproduction in any medium, provided the original work is properly cited. regarding the etiology, outcomes, prevention, and cure of the disease [1]. The pressing issue is that fake news spreads more rapidly on social media than news from reliable sources, damaging the authenticity balance of the news ecosystem. A recent analysis showed that during the 2016 presidential election in the United States, fake and biased news was more outspread than news from 19 authentic sources combined [2]. This issue is becoming a public health concern as exposure to a high volume of information can lead to media fatigue, causing the discontinuation of healthy behaviors that are essential to protect individuals. Furthermore, misinformation and rumors regarding COVID-19 are hindering the practice of healthy behaviors (such as handwashing and social distancing) and promoting erroneous practices that increase the spread of the virus and ultimately result in poor physical and mental health outcomes. For example, in India, a father of three was reported 
to commit suicide upon learning that he had been diagnosed with COVID-19 [3]. Another example of hazards attributable to improper health communication can be drawn from Nigeria, where the health officials found several cases of overdose of chloroquine (a drug used to treat malaria) after news on the purported effectiveness of the drug for treating COVID-19 spread through the news media [4]. Rumors about a national lockdown in the United States fueled panic buying of groceries and paper products, which disrupted the supply chain and exacerbated demand-supply gaps and food insecurity among individuals with low socioeconomic status and other vulnerable populations [5]. Similar phenomena have been observed in other economies around the world, which may have prolonged impacts on public health nutrition and psychosocial health among the affected populations. The widespread misinformation related to the diagnosis and treatment of COVID-19 has confused both the general population and healthcare providers, who are battling a relatively under-researched disease. This phenomenon is also reducing the legitimacy of new scientific discoveries regarding potential cures or vaccine candidates for this disease. These hoaxes and rumors are also creating a social stigma around COVID-19, which has resulted in reduced compliance with home quarantine and social isolation. Several countries have reported incidents where hundreds of individuals were infected by a single person who visited their mosque/church despite their doctor's advice to remain isolated at home [6]. Such problems are contributing to the suboptimal control of the COVID-19 pandemic across various populations.

It is understandable that people living in quarantine, isolation, or at risk of an infectious disease outbreak are likely to experience psychosocial stress and adverse health outcomes, which may provoke their interest in learning more about the disease. However, such situations require assurance complemented by a flow of correct information. This need has been acknowledged by the World Health Organization, which has partnered with several social media platforms and seven major tech companies - namely, Facebook, Google, Linkedln, Microsoft, Reddit, Twitter, and YouTube-that agreed to stamp out fraud and misinformation, and to promote critical updates from healthcare agencies [7]. Despite these agreements, rumors are still rampant on all these platforms, a problem that necessitates several multipronged interventions to address. First, frontline healthcare providers should be equipped with the most recent research findings and accurate information, which can be used for direct caregiving and communicated to at-risk patients or populations. Doing so may also facilitate more robust patient-provider communication and result in better health outcomes. Previously, the integration of information technology successfully improved patient care in managing mass casualty incidents, yielding several positive outcomes in the form of optimization of resources, extension of care, and improved health outcomes of patients [8]. Secondly, mass media, community organizations, support groups, and civil society may play critical roles in disseminating accurate information. To achieve this, it is necessary to build strategic partnerships at local and global levels, connecting offline and online resources in a coordinated manner so that validated information is communicated across platforms. Third, all hoaxes and rumors should be removed from all online platforms, and only scientifically sound information should be allowed to improve awareness among the general population. Advanced technologies like natural language processing or text-mining approaches may be helpful in detecting online content that has no scientific basis. The latest data-mining algorithms are successfully detecting the unique characteristics of all kinds of fake news and removing them from their respective platforms [2]. Twitter, Reddit, and Amazon have already implemented these methods to remove fake accounts or product reviews. Application of the same principles to remove all rumors and hoaxes regarding this pandemic is therefore evidently feasible, despite its unique challenges. Therefore, social media and other online providers should adopt such measures to identify and eliminate potentially harmful misinformation and rumors. Fourth, online portals and personnel involved with the production and propagation of such misinformation should be brought to justice. Local and national regulatory authorities and law enforcement agencies should be made aware of these challenges in order for them to address these issues comprehensively in their respective contexts. In this regard, the governments of many countries have arrested individuals involved with the spread of such rumors [9]. However, before enforcing any of these laws worldwide, authorities should conduct a proper investigation and ensure the legal rights of the accused. Fifth, common platforms should be launched to provide precautionary information in both online and offline media at a large scale. Successful strategies for mass media campaigns from the last few epidemics should be identified and implemented. These messages should be culturally tailored and translated to several languages to increase outreach. For example, media coverage of the recent severe acute respiratory syndrome, H1N1 
influenza, Middle East respiratory syndrome, Ebola, and H7N9 influenza epidemics had a significant effect on reducing the total spread of these pathogens by promoting healthy behaviors [10]. Factual information regarding COVID-19 can also be expected to have similar results. Sixth, telemedicine should be used to provide evidence-based care and informational services should be made available for people with limited access to care, such as people in rural areas, the elderly, and those who are simply stuck at home due to lockdown. This will both decrease the risk of hospital-acquired infections and also reduce the utilization of resources such as ventilators and personal protective equipment for doctors. Lastly, transparency, good information governance, and leadership across communities and institutions should be promoted to remove misleading information related to COVID-19, which is essential for the greater good of populations experiencing the health and psychosocial burdens of this pandemic.

\section{Ethics Statement}

This paper is a perspective so it did not need ethical consideration.

\section{CONFLICT OF INTEREST}

The authors have no conflicts of interest associated with the material presented in this paper.

\section{FUNDING}

None.

\section{ACKNOWLEDGEMENTS}

None.

\section{AUTHOR CONTRIBUTIONS}

Conceptualization: ST. Data curation: MMH, HM. Formal analysis: MMH, HM. Funding acquisition: None. Writing - original draft: ST. Writing - review \& editing: MMH, HM.

\section{ORCID}

Samia Tasnim https://orcid.org/0000-0002-2686-1840 Md Mahbub Hossain https://orcid.org/0000-0002-7059-
7768

Hoimonty Mazumder https://orcid.org/0000-0003-37873780

\section{REFERENCES}

1. Raman S. Public health experts worry about spread of COVID-19 misinformation: some say federal agencies should do more to stamp out misconceptions; 2020 Mar 18 [cited 2020 Mar 28]. Available from: https://www.rollcall.com/2020/03/18/publichealth-experts-worry-about-spread-of-covid-19-misinformation/.

2. Shu K, Sliva A, Wang S, Tang J, Liu H. Fake news detection on social media: a data mining perspective. SIGKDD Explor 2017; 19(1):22-36.

3. Wallen J. Coronavirus: Indian man 'died by suicide' after becoming convinced he was infected. Telegraph; 2020 Feb 12 [cited 2020 Mar 28]. Available from: https://www.telegraph. co.uk/global-health/science-and-disease/coronavirus-indianman-died-suicide-becoming-convinced-infected/.

4. Busari S, Adebayo B. Nigeria records chloroquine poisoning after Trump endorses it for coronavirus treatment. CNN; 2020 Mar 23 [cited 2020 Mar 28]. Available from: https://edition. cnn.com/2020/03/23/africa/chloroquine-trump-nigeria-int// index.html.

5. Spencer SH. False claims of nationwide lockdown for COVID-19. Factcheck; 2020 Mar 16 [cited 2020 Mar 28]. Available from: https://www.factcheck.org/2020/03/false-claims-of-nationwide-lockdown-for-covid-19/.

6. Kasulis K. 'Patient 31' and South Korea's sudden spike in coronavirus cases. Aljazeera; 2020 Mar 3 [cited 2020 Mar 28]. Available from: https://www.aljazeera.com/news/2020/03/31-southkorea-sudden-spike-coronavirus-cases-200303065953841. html?xif = .

7. Statt N. Major tech platforms say they're 'jointly combating fraud and misinformation' about COVID-19. Verge; $2020 \mathrm{Mar}$ 16 [cited 2020 Mar 28]. Available from: https://www.theverge. com/2020/3/16/21182726/coronavirus-covid-19-facebookgoogle-twitter-youtube-joint-effort-misinformation-fraud.

8. Zhao X, Rafiq A, Hummel R, Fei DY, Merrell RC. Integration of information technology, wireless networks, and personal digital assistants for triage and casualty. Telemed J E Health 2006; 12(4):466-474.

9. HK police arrest man "for spreading fake virus news". RTHK News; 2020 Feb 4 [cited 2020 Mar 28]. Available from: https:// 
news.rthk.hk/rthk/en/component/k2/1506616-20200204. htm.

10. Collinson S, Khan K, Heffernan JM. The effects of media reports on disease spread and important public health measurements. PLoS One 2015;10(11):e0141423. 